\title{
Predicting Electrical Cardioversion Outcome from Surface ECG Recordings Through Wavelet Sample Entropy
}

\author{
R Alcaraz ${ }^{1}$, JJ Rieta ${ }^{2}$ \\ ${ }^{1}$ Innovation in Bioengineering Research Group. University of Castilla-La Mancha, Cuenca, Spain \\ ${ }^{2}$ Biomedical Synergy. Valencia University of Technology, Valencia, Spain
}

\begin{abstract}
Electrical Cardioversion (ECV) is the most effective alternative to revert persistent atrial fibrillation $(P A F)$ back to normal sinus rhythm (NSR). It would be clinically useful to predict NSR maintenance likelihood after ECV before it is attempted because of the high atrial fibrillation $(A F)$ risk of recurrence and because of the potential secondary effects of ECV. In previous studies, other parameters have been deeply analyzed, but their results were very different and, consequently, inconclusive. Thereby, this work presents a new non-invasive predictor of ECV outcome before it is attempted. This method is based on the suitable combination of the Wavelet Transform (WT) and sample entropy (SampEn), which is a non-linear regularity index, and is called Wavelet Sample Entropy (WSE). Results indicated that 17 out of 21 (80.95\%) ECVs relapsing to AF and 12 out of 14 (85.71\%) ECVs resulting in NSR after the first month were correctly discriminated. The recordings that relapsed to AF presented higher SampEn values $(0.0320$ $\pm 0.0053)$ than the recordings in NSR $(0.0271 \pm 0.0045)$. In addition, the five patients in which NSR was not restored in any case, presented the highest SampEn values (0.0350 $\pm 0.0028)$.
\end{abstract}

\section{Introduction}

Electrical cardioversion (ECV) is one common therapy in the treatment of persistent (not self-terminated) atrial fibrillation (AF) patients. In contrast to pharmacological cardioversion, $\mathrm{ECV}$ is the most effective alternative to revert persistent AF back to normal sinus rhythm (NSR), especially if the arrhythmia has been present for more than 24 hours [1]. Although the ECV success rate is high, $\mathrm{AF}$ recurrence is common, especially during the first 2 weeks following the procedure [2]. Moreover, ECV also has the potential of causing severe collateral effects, such as post-shock bradycardia, malignant ventricular arrhythmias, arterial thromboembolism and complications related to anaesthesia [1]. Hence, it would be clinically very use- ful to predict NSR maintenance after ECV, before it is attempted. In this way, the risks of cardioversion could be avoided for those patients with high risk of short-term recurrence and, for the health care provider, clinical costs could be optimized because unproductive treatment time and bed usage could be reduced.

On the other hand, Wavelet Transform (WT) has been successfully employed to solve several problems dealing with electrocardiogram (ECG) signals, such as feature extraction and discrimination between normal and abnormal beats [3], detection of ventricular late potentials [4], evaluation of instantaneous changes in heart rate variability [5], screening of patients with congestive heart failure [6], etc.

Hence, in this work, wavelet analysis was combined with a regularity study to predict non-invasively ECV outcome before it is attempted. This study was performed through the application of Sample Entropy (SampEn), which is a non-linear index for quantifying time series regularity [7]. The objective was to evaluate the atrial activity $f$ waves regularity time course. In this respect, structural changes into $f$ waves could reflect the AF organization variation. The analysis of this variation is crucial, because some works have suggested that NSR maintenance would be more likelihood in patients who present a highly organized AF, because the more disorganized the AF, the higher the number of propagating wavelets [8], and the larger the atrial volume that could support reentries propagation after the shock [9].

\section{Materials}

Forty patients (15 men and 25 women) with persistent AF lasting more than 30 days, undergoing the first attempt of ECV were followed during four weeks. All patients were in drug treatment with amiodarone. A standard 12lead ECG was acquired prior to cardioversion. All signals were digitized at a sampling rate of $1024 \mathrm{~Hz}$ and 16-bit resolution with a Cardiolab System in the electrophysiology laboratory during ECV protocol. In order to process these signals, a 30 seconds-length AF segment preceding the ECV was extracted for each patient. After the ECV, 
NSR was not restored in 5 patients $(12.5 \%)$ whereas in 21 $(52.5 \%)$ NSR duration was below one month. In the remaining 14 patients $(35 \%)$ NSR was maintained.

Lead $V_{1}$ was chosen for the analysis because previous works have shown that atrial activity (AA) is dominant in this lead [10]. The signal was preprocessed in order to improve later analysis. Firstly, baseline wander was removed making use of bidirectional high pass filtering with $0.5 \mathrm{~Hz}$ cutt-off frequency [11]. Secondly, high frequency noise was reduced with an eight order bidirectional IIR Chebyshev low pass filtering, whose cut-off frequency was $70 \mathrm{~Hz}$ [12]. Finally, powerline interference was removed through adaptive notch filtering, which preserves the ECG spectral information [13].

\section{Methods}

\subsection{Wavelet transform}

The Continuous Wavelet Tranform (CWT) is defined as the sum over all time of the signal $f(t)$ scaled with a factor $a$ and shifted a certain time interval $b$ through the mother wavelet function $\Psi[14]$ :

$$
\begin{gathered}
C(a, b)=\int_{-\infty}^{\infty} f(t) \cdot \Psi_{a, b}(t) d t \\
\Psi_{a, b}(t)=\frac{1}{\sqrt{a}} \Psi\left(\frac{t-b}{a}\right) \quad \forall a, b \in \Re^{+}
\end{gathered}
$$

The results of the CWT are many wavelet coefficients $C$, which are a function of scale and position. Concretely, a wavelet coefficients vector is obtained for each analyzed scale [14]. Discrete time Wavelet Transform (DWT) is the sampled version of the CWT in a dyadic grid. One advantage of the DWT is that the original signal can be synthesized using fewer coefficients than in the case of the CWT. An efficient way to implement the DWT using filters was developed by Mallat, and was called multiresolution algorithm [15].

\subsection{Sample entropy}

Sample Entropy (SampEn) examines time series for similar epochs and assigns a non-negative number to the sequence, with larger values corresponding to more complexity or irregularity in the data [7]. Two input parameters, a run length $m$ and a tolerance window $r$, must be specified for SampEn to be computed. $\operatorname{SampEn}(m, r)$ is the negative logarithm of the conditional probability that two sequences similar during $m$ points remain similar at the next point, where self-matches are not included in calculating the probability. A detailed mathematic description can be found in [7].

Although $m$ and $r$ are critical in determining the outcome of SampEn, no guidelines exist for optimizing their values. Nevertheless, the $m$ and $r$ values suggested by Pincus are $m=1$ or $m=2$ and $r$ between 0.1 and 0.25 times the standard deviation of the original time serie [16].

\subsection{Wavelet sample entropy}

The analysis of the AA from the surface ECG is complicated by the simultaneous presence of ventricular activity, which is of much greater amplitude. Whereby, the AA signal has to be firstly extracted before the application of any other analysis. Although a variety of different techniques exist for this purpose, a QRST cancellation method was used. Thus, the highest variance eigenvector of all the ECG beats was considered as the ventricular template for the cancellation. This QRST template was selected because it was able to obtain a more accurate ventricular activity representation and, hence, higher quality AA extraction in short AF recordings, such as the analyzed in this work, than those obtained by averaging all the beats [17].

Next, eight levels of wavelet decomposition were applied to the AA signals because the seventh detail scale (sub-band corresponding to 4-8 Hz) covers the most typical AA frequency range [18]. Regarding the wavelet family selection, there are no established rules for the choice of wavelet functions. Thereby, several orthogonal wavelet families were tested, because only in an orthogonal basis any signal can be uniquely decomposed and the decomposition can be inverted without loosing information [19]. The best results were obtained by applying the Biorthogonal wavelet family of order 4.4 and, hence, this wavelet function was selected.

The wavelet coefficients vector corresponding to the scale containing the dominant atrial frequency, those with the largest amplitude within the AA frequency range [18], was linearly interpolated by the factor $2^{m-1}$, being $m$ the discrete wavelet scale. Hence, a vector of wavelet coefficients with a number of samples equal to the original signal was obtained for the chosen scale. Considering that different scales present wavelet coefficients vectors with different number of samples, this interpolation was necessary. Moreover, unsuccessful results were obtained when noninterpolated wavelet coefficients vectors were analyzed. Finally, the regularity of this vector was estimated making use of SampEn to discern between ECVs relapsing to AF and resulting in NSR. This combination of WT and SampEN has been named Wavelet Sample Entropy (WSE) and allow us to detect regularity variations in the AA signal that would be left masked in other cases.

\subsection{Statistical analysis}

Results were expressed as mean \pm standard deviation, unless otherwise specified. In order to evaluate the organization analysis predictive ability for the NSR maintenance, 
receiver operating characteristic (ROC) curves were constructed. Different thresholds or cutoff points (SampEn values) were selected and the sensitivity/specificity pair for each one of them was calculated. Sensitivity (the true positive rate) is the ECVs relapsing to AF proportion correctly classified (SampEn value higher than the cutoff point), whereas specificity (the true negative rate) represents the ECVs resulting in NSR percentage correctly recognized (SampEn value lower than the cutoff point). The closest point to $100 \%$ sensitivity and specificity was selected as optimum SampEn threshold. The $t$ Student test was used to determine whether there was any significant difference between the groups. A two-tailed value of $p<0.05$ was considered statistically significant.

\section{Results}

The ROC curve provided 0.0301 as optimum SampEn discrimination threshold, in which $80.95 \%$ (17 out of 21 ) sensitivity and $85.71 \%$ (12 out of 14) specificity were obtained, see Fig. 1(a). Therefore, the ECV outcome in 29 out of 35 patients $(82.86 \%)$ were correctly predicted. An AROC of 0.882 was achieved. Moreover, the patients relapsing to AF presented higher SampEn values (0.0320 $\pm 0.0053)$ than those resulting in NSR after one month $(0.0271 \pm 0.0045)$, such as Fig. 1(b) shows. Also, both groups were statistically distinguishable, since a statistic significance lower than 0.001 was obtained.

In addition, the five patients who relapsed to AF immediately after the ECV were also studied. For this case, the methodology provided the highest SampEn values (0.0350 $\pm 0.0028)$, thus reinforcing the obtained results consistency.

\section{Discussion and conclusions}

The presence of more structured $f$ waves in organized atrial activities [10] could justify the obtained results, which show that patients who relapsed to AF presented lower wavelet coefficients vector regularity than those who remained in NSR. In persistent AF, the presence of structured $f$ waves suggests a more organized AA and, hence, the obtained results agree with the findings reported in other works, such as: (i) the higher the AA organization, the higher the success rates in AF cardioversion [20, 8], (ii) the higher the AA organization, the lower the energy required for successful cardioversion [9] and (iii) paroxysmal AF requires less energy for cardioversion than persistent AF [21].

In addition, the five patients in which NSR was not immediately restored after ECV presented the wavelet coefficients vectors with the lowest regularities. Thus, obtained results consistency is reinforced and it can be concluded that in those patients with more organized $f$ waves the AF recurrence likelihood is lower after ECV.

Several clinical, electrophysiological and demographic features (AF duration, type of underlying disease, patient age, left atrium diameter, etc.) have been proposed in previous works as predictors for successful AF cardioversion and sinus rhythm maintenance. But the predictive value of these parameters is far from optimal [22]. On the other hand, conflicting results were obtained when the predictive ability of atrial fibrillatory rate was studied in patients with long-duration AF. Some authors [22] suggested that the confounding effect of antiarrhythmic drug therapy could explain the differences among these results, however, this aspect still remains unclear [23]. Hence, the results obtained with WSE can be considered as very promising. Nevertheless, the number of analyzed cardioversions has been reduced and, therefore, the presented results must be considered with caution. Moreover, a wide data set allowing a more rigorous statistical analysis is required to provide confidence in the robustness of the method.

\section{Acknowledgements}

This work was partly supported by the projects PAID04-08 and TEC2007-64884 from the Spanish Ministry of Education and Science.

\section{References}

[1] Gall NP, Murgatroyd FD. Electrical cardioversion for AFthe state of the art. Pacing Clin Electrophysiol Apr 2007; 30(4):554-567.

[2] Tieleman RG, Gelder ICV, Crijns HJ, Kam PJD, Berg MPVD, Haaksma J, Woude HJVD, Allessie MA. Early recurrences of atrial fibrillation after electrical cardioversion: a result of fibrillation-induced electrical remodeling of the atria? J Am Coll Cardiol Jan 1998;31(1):167-173.

[3] Matsuyama A, Jonkman M, de Boer F. Improved ECG signal analysis using wavelet and feature extraction. Methods Inf Med 2007;46(2):227-230.

[4] Vai MI, Zhou LG. Beat-to-beat ECG ventricular late potentials variance detection by filter bank and wavelet transform as beat-sequence filter. IEEE Trans Biomed Eng Aug 2004; 51(8):1407-1413.

[5] Belova NY, Mihaylov SV, Piryova BG. Wavelet transform: A better approach for the evaluation of instantaneous changes in heart rate variability. Auton Neurosci Jan 2007; 131(1-2):107-122.

[6] Hossen A, Al-Ghunaimi B. A wavelet-based soft decision technique for screening of patients with congestive heart failure. Biomedical Signal Processing and Control 2007; 2:135-143.

[7] Richman JS, Moorman JR. Physiological time-series analysis using approximate entropy and sample entropy. Am J Physiol Heart Circ Physiol Jun 2000;278(6):H2039H2049. 


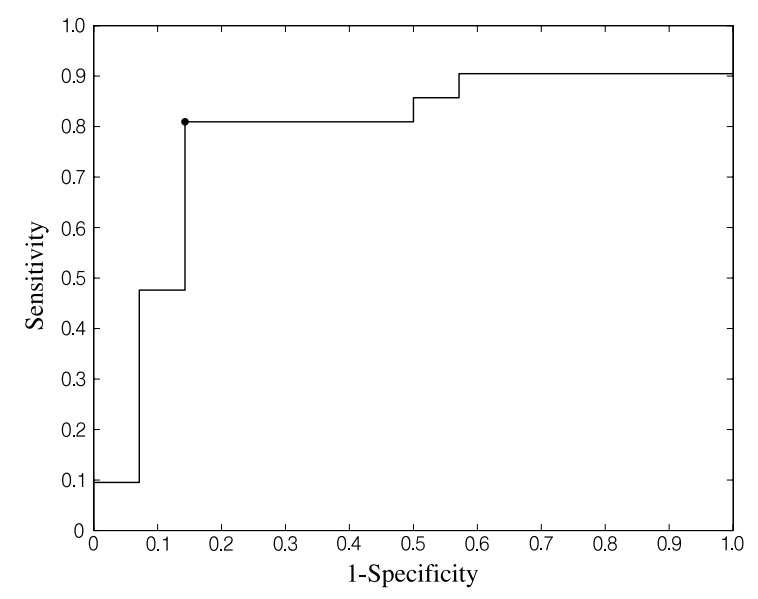

(a)

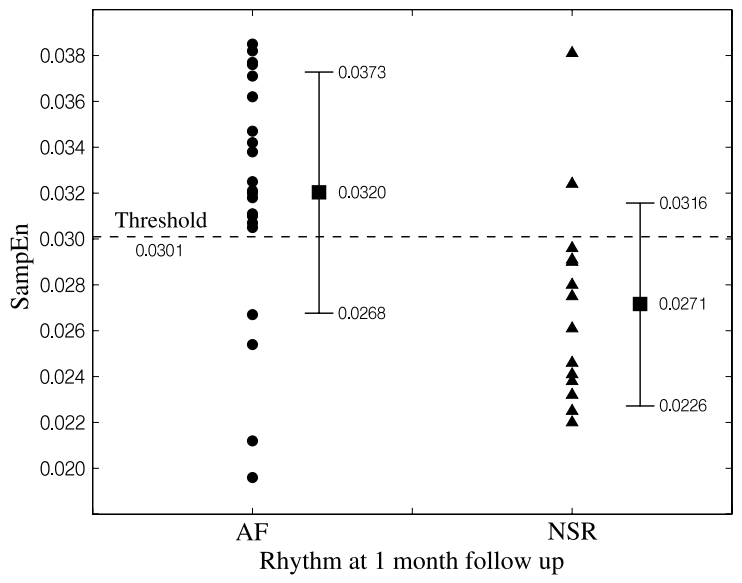

(b)

Figure 1. (a) ROC curve constructed with the obtained SampEn values for persistent AF patients. The closest point to $100 \%$ sensitivity and specificity is selected as optimum SampEn threshold. Symbol $\bullet$ indicates the optimum threshold. (b) Classification into patients resulting in NSR and relapsing to AF after 4 weeks following ECV.

[8] Sih HJ, Zipes DP, Berbari EJ, Olgin JE. A high-temporal resolution algorithm for quantifying organization during atrial fibrillation. IEEE Trans Biomed Eng Apr 1999; 46(4):440-450.

[9] Calcagnini G, Censi F, Michelucci A, Bartolini P. Descriptors of wavefront propagation. Endocardial mapping of atrial fibrillation with basket catheter. IEEE Eng Med Biol Mag 2006;25(6):71-78.

[10] Petrutiu S, Ng J, Nijm GM, Al-Angari H, Swiryn S, Sahakian AV. Atrial fibrillation and waveform characterization. A time domain perspective in the surface ECG. IEEE Eng Med Biol Mag 2006;25(6):24-30.

[11] Dotsinsky I, Stoyanov T. Optimization of bi-directional digital filtering for drift suppression in electrocardiogram signals. J Med Eng Technol 2004;28(4):178-180.

[12] Sun Y, Chan K, Krishnan SM. ECG signal conditioning by morphological filtering. Comput Biol Med Nov 2002; 32(6):465-479.

[13] Ferdjallah M, Barr RE. Adaptive digital notch filter design on the unit circle for the removal of powerline noise from biomedical signals. IEEE Trans Biomed Eng Jun 1994; 41(6):529-536.

[14] Addison PS. The Illustrated Wavelet Transform Handbook. Introductory Theory and Applications in Science, Engineering, Medicine and Finance. Institute of Physics Publishing, 2002. ISBN 0-7503-0692-0.

[15] Mallat SG. A theory for multiresolution signal decomposition: The wavelet representation. IEEE Trans Pattern Anal Mach Intell 1989;11(7):674-693. ISSN 0162-8828.

[16] Pincus SM. Approximate entropy as a measure of system complexity. Proc Natl Acad Sci U S A Mar 1991; 88(6):2297-2301.

[17] Alcaraz R, Rieta JJ. Adaptive singular value QRST cancellation for the analysis of short single lead atrial fibrillation electrocardiograms. Proc Comput Cardiol 2007;34:513516.

[18] Bollmann A, Husser D, Mainardi L, Lombardi F, Langley P, Murray A, Rieta JJ, Millet J, Olsson SB, Stridh M, Sörnmo L. Analysis of surface electrocardiograms in atrial fibrillation: techniques, research, and clinical applications. Europace Nov 2006;8(11):911-926.

[19] Mallat S. A Wavelet Tour of Signal Processing. Academic Press, 1999. ISBN 0-12-466606-X.

[20] Everett TH, Kok LC, Vaughn RH, Moorman JR, Haines DE. Frequency domain algorithm for quantifying atrial fibrillation organization to increase defibrillation efficacy. IEEE Trans Biomed Eng Sep 2001;48(9):969-978.

[21] Lau CP, Lok NS. A comparison of transvenous atrial defibrillation of acute and chronic atrial fibrillation and the effect of intravenous sotalol on human atrial defibrillation threshold. Pacing Clin Electrophysiol Oct 1997;20(10 Pt 1):2442-2452.

[22] Pálinkás A, Antonielli E, Picano E, Pizzuti A, Varga A, Nyúzó B, Alegret JM, Bonzano A, Tanga M, Coppolino A, Forster T, Baralis G, Delnevo F, Csanády M. Clinical value of left atrial appendage flow velocity for predicting of cardioversion success in patients with non-valvular atrial fibrillation. Eur Heart J Dec 2001;22(23):2201-2208.

[23] Holmqvist F, Stridh M, Waktare JEP, Sörnmo L, Olsson SB, Meurling CJ. Atrial fibrillatory rate and sinus rhythm maintenance in patients undergoing cardioversion of persistent atrial fibrillation. Eur Heart J Sep 2006;27(18):2201-2207.

Address for correspondence:

Raúl Alcaraz Martínez

E. U. Politécnica de Cuenca

Campus Universitario

16071 Cuenca (Spain)

raul.alcaraz@uclm.es 